\title{
The Pierre Auger Observatory Upgrade
}

\author{
Giovanni Marsella ${ }^{1,2, a}$ for the Pierre Auger Collaboration ${ }^{b}$ \\ ${ }^{1}$ Universitá degli Studi del Salento \\ ${ }^{2}$ INFN sez. Lecce
}

\begin{abstract}
It is planned to operate the Pierre Auger Observatory until at least the end of 2024. An upgrade of the experiment has been proposed in order to provide additional measurements to allow one to elucidate the mass composition and the origin of the flux suppression at the highest energies, to search for a flux contribution of protons up to the highest energies and to reach a sensitivity to a contribution as small as $10 \%$ in the flux suppression region, to study extensive air showers and hadronic multi-particle production. With operation planned until 2024, event statistics will more than double compared with the existing Auger data set, with the critical added advantage that every event will now have mass information. Obtaining additional composition-sensitive information will not only help to better reconstruct the properties of the primary particles at the highest energies, but also improve the measurements in the energy range just above the ankle. Furthermore, measurements with the new detectors will help to reduce systematic uncertainties related to the modelling hadronic showers and to limitations in the reconstruction algorithms.

A description of the principal proposed Auger upgrade will be presented. The Auger upgrade promises high-quality future data, and real scope for new physics.
\end{abstract}

\section{Introduction}

Since its starting time, the Pierre Auger Observatory [1] gave a strong contribution to the understanding of ultra high-energy cosmic rays (UHECRs). The principal motivations of the proposed AUGER upgrade [2] is to provide additional measurements to allow the collaboration to address the following issues:

- The origin of the flux suppression at the highest energies and the measurement of the mass composition beyond the reach of the Fluorescence Detector (FD).

- Search for a proton contribution in the flux suppression region $\left(E>5 \times 10^{19} \mathrm{eV}\right)$, aiming at reaching a sensitivity to a proton contribution as small as $10 \%$ in the flux suppression region, search of point sources and estimate the physics potential of existing and future cosmic ray, neutrino, and gamma-ray detectors.

- Fundamental particle physics at energies beyond man-made accelerators and studies of extensive air showers and hadronic multiparticle production.

\footnotetext{
a e-mail: giovanni.marsella@le.infn.it

${ }^{b}$ Full author list: http://auger.org/archive/authors_2016_06.html
} 
The proposed upgrade will consist in: (i) the addition of a plastic scintillator plane (SSD) above the existing Water-Cherenkov Detectors (WCD); (ii) the installation in the Surface Detector (SD) stations of new electronics that will process both WCD and SSD signals; (iii) the installation of an Underground Muon Detector (UMD) in the existing SD infill area; (iv) a change in the operation mode of the Fluorescence Detector (FD) to extend measurements into periods with higher night sky background.

\section{Surface Scintillator Detector}

The key element of the upgrade will be the installation of a new detector consisting of a plastic scintillator plane above each of the existing water-Cherenkov detectors. This scintillation detector will provide a complementary measurement of the shower particles: they will be sampled with two detectors having different responses to muons and electromagnetic particles, allowing for the reconstruction of the different shower components. The design of the surface scintillator detectors (SSDs) is simple and reliable. The SSD unit will consist of a box of $3.8 \mathrm{~m} \times 1.3 \mathrm{~m}$, housing two scintillator modules, each covering an area of $1.9 \mathrm{~m}^{2}$, see Fig. 1 (left). They will be easily deployed over the full $3000 \mathrm{~km}^{2}$ area of the overall Auger Surface Detector (SD). The $10 \mathrm{~mm}$ thick scintillators are read out by wavelength-shifting fibers guiding the light of the two modules to a PMT. The response over the area of the scintillator is uniform within 5\%. In Fig. 1 (right) the charge distribution of signals in the SSD triggered by coincidences in the larger WCD is shown. A clean separation of Minimum Ionizing Particle (MIP) signals from the background is evidenced. An engineering array of 10 detectors has been installed at the Auger site since September 2016.
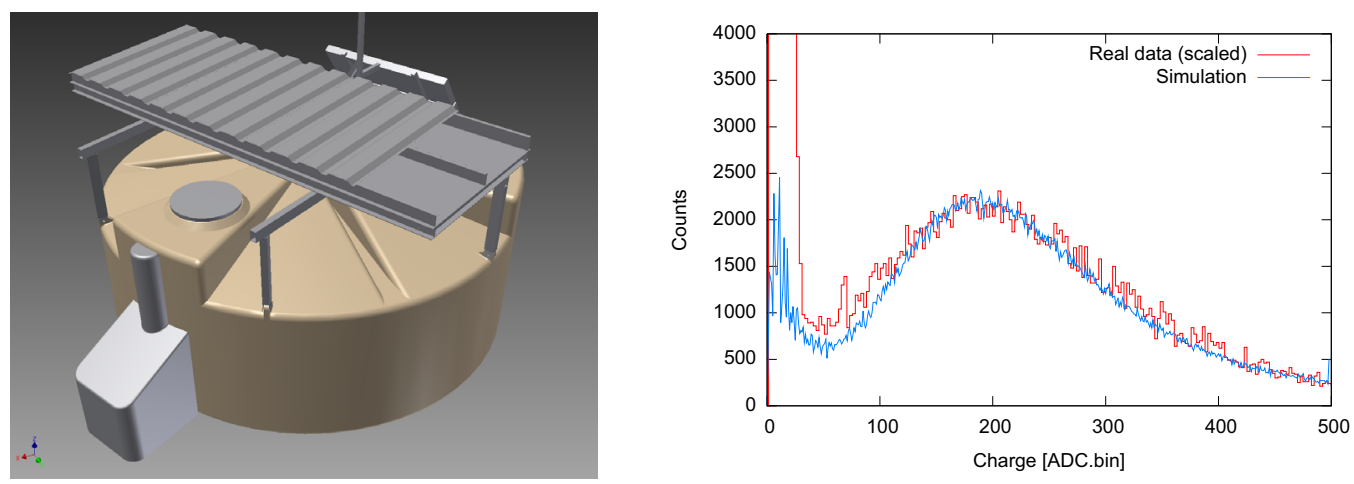

Figure 1. Left panel: 3D view of a SSD mounted on a WCD. A double roof, with the upper layer being corrugated aluminum is used to reduce the temperature variations. Right panel: Charge distribution for Minimum Ionizing Particles taken with a $2 \mathrm{~m}^{2}$ prototype installed in the Auger array. The data correspond to one minute of data taking and are well reproduced by the detector simulation based on Geant 4 .

\section{Surface Detector Upgrade}

The surface detector stations will be upgraded with new electronics that will process both WCD and SSD signals. It will increase the data quality with faster sampling of ADC traces $(120 \mathrm{MHz})$, giving a better timing accuracy, and increased dynamic range (12 bits). To enhance the local trigger and 
processing capabilities a more powerful local station processor and FPGA have been implemented. This also improves calibration and monitoring capabilities of the surface detector stations. The surface detector electronics upgrade (SDEU) can be easily deployed, and will have only minimal impact on the continuous data taking of the Surface Detector. To increase the dynamic range also a 4th PMT of 1 " of diameter, corresponding to 1/9 of of the WCD PMTs, will be added. With this solution the total dynamic range of the SD will correspond to 22 effective ADC bits, ranging from fractions of VEM (energy from Vertical Equivalent Muon) to 20000 VEM. This range is due to the 3 different ADC channels, each 12 bits wide and shifted by 5 bits corresponding to the relative gain, of the $30 \mathrm{~dB}$ amplified SD standard PMT, the OdB SD standard PMT and the small PMT respectively. This leads to less than $2 \%$ saturated events at the highest energies and unambiguous determination of the particle density down to less than $300 \mathrm{~m}$ from the shower core.

\section{Underground Muon Detector}

An Underground Muon Detector (UMD) is being installed in the existing SD infill area of $23.5 \mathrm{~km}^{2}$. The UMD will provide important direct measurements of the shower muon content and its time structure. It will also serve as a verification and fine-tuning of the methods used to extract the muon information with the SSD and WCD measurements. The performance and characteristics of the AMIGA project [3] match these requirements, and thus the completed AMIGA array will serve as the UMD. 61 muon detectors $\left(30 \mathrm{~m}^{2}\right)$ are planned and will be deployed on a $750 \mathrm{~m}$ spaced grid. The muon detectors are shielded by $1.3 \mathrm{~m}$ of soil.

\section{Fluorescence Detector Upgrade}

The Fluorescence Detector [4] provides exceptional information such as model-independent energy reconstruction and mass composition measurement. The main limitation of the FD is its duty cycle that nowadays reaches $15 \%$. The current criteria for FD measurement are:

- The sun more than $18^{\circ}$ below the horizon

- The moon remains below horizon for longer than 3 hours

- The illuminated fraction of the moon must be below $70 \%$

The operation mode of the Fluorescence Detector (FD) will be changed to extend measurements into periods with higher night sky background by lowering the PMT HV supply thus reducing the PMT gain by a factor of ten. A successful test has already been done over a period of seventeen nights. This will allow to increase the current duty cycle of the FD by about $50 \%$.

The combination of SD and FD upgrades are also expected to increase the sensitivity to the flux of photons and neutrinos (see Fig. 2).

\section{Conclusions}

The AUGER upgrade will allow to study the mass composition above $5 \times 10^{19} \mathrm{eV}$. The different proposed models, GZK energy loss model versus a picture defining the maximum energy of sources, will be analyzed with a higher and more precise statistics. The sensitivity to the proton flux above $5 \times 10^{19} \mathrm{eV}$ will be enhanced to reach a sensitivity to a contribution as small as $10 \%$. Finally the upgrade will enhance the possibility of studying new particle physics beyond the reach of LHC, due to the combination of detectors allowing for a better particle identification. Actually 12 Engineering 

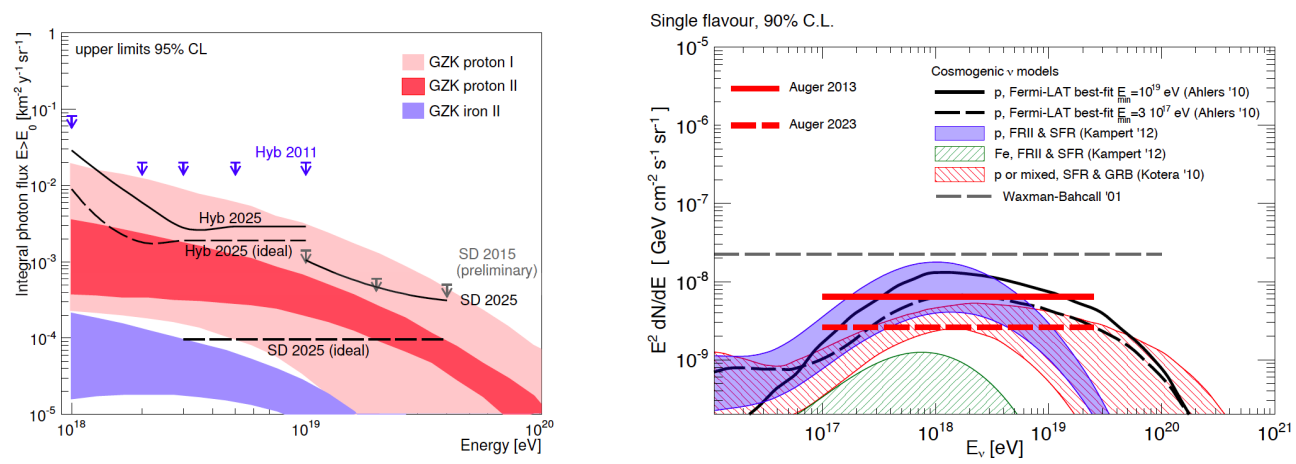

Figure 2. Expected sensitivity on the flux of photons and neutrinos. In addition to the conservative estimates based on the increase of statistics, also the projected photon sensitivity for the ideal case of being able to reject any hadronic background due to the upgraded surface detector array is shown [2] .

Array stations have been installed and are starting taking data. In early 2017 the detector evaluation will be completed and at the beginning of 2018 the deployment of the AUGER upgrade will start. The detector is expected to take data at least until 2024 with an exposure up to $40000 \mathrm{~km}^{2} \mathrm{sr}$ yr, allowing to collect a statistics comparable with the one reached so far by the AUGER experiment.

\section{References}

[1] Pierre Auger Collaboration, A. Aab et al., The Pierre Auger Cosmic Ray Observatory, Nucl. Instrum. Meth. A 798, 172-213 (2015) [http://arxiv.org/abs/1502.013231502.01323].

[2] Pierre Auger Collaboration, A. Aab et al., The Pierre Auger Observatory Upgrade, [http://arxiv.org/abs/1604.036371604.03637].

[3] Pierre Auger Collaboration, B. Wundheiler et al., The AMIGA Muon Counters of the Pierre Auger Observatory: Performance and Studies of the Lateral Distribution Function, Proc. of 34th Int. Cosmic Ray Conf., The Hague, PoS(ICRC2015)324 (2015).

[4] Pierre Auger Collaboration, J. Abraham et al., The Fluorescence Detector of the Pierre Auger Observatory, Nucl. Instrum. Meth. A620 (2010) 227-251, [http://arxiv.org/abs/0907.42820907.4282]. 